\title{
Enzyme kinetics for a two-step enzymic reaction with comparable initial enzyme-substrate ratios
}

\author{
C. L. Frenzen ${ }^{1}$ and P. K. Maini ${ }^{2}$ \\ 1 Department of Mathematics, Southern Methodist University, Dallas, TX 75275, USA \\ ${ }^{2}$ Centre for Mathematical Biology, Mathematical Institute, 24-29 St. Giles, Oxford OX1 3LB, UK
}

\begin{abstract}
We extend the validity of the quasi-steady state assumption for a model double intermediate enzyme-substrate reaction to include the case where the ratio of initial enzyme to substrate concentration is not necessarily small. Simple analytical solutions are obtained when the reaction rates and the initial substrate concentration satisfy a certain condition. These analytical solutions compare favourably with numerical solutions of the full system of differential equations describing the reaction. Experimental methods are suggested which might permit the application of the quasi-steady state assumption to reactions where it may not have been obviously applicable before.
\end{abstract}

Key words: Michaelis Menten approximation - Quasi-steady state assumption - Scaling -- Singular perturbations - Fast and slow timescales

\section{Introduction}

Many biochemical reactions exhibit an initial fast transient followed by a slowly varying change in reactant concentration. A firmly established mathematical technique for dealing with the system of ordinary differential equations describing such reactions is the quasi-steady state assumption (QSSA) or pseudo-steady state hypothesis. Essential to this technique is the isolation of a small parameter to enable singular perturbation methods to be employed. In enzyme substrate kinetics this small parameter is usually taken to be the ratio of the initial enzyme to substrate concentration (Michaelis-Menten [1]). A discussion of the mathematical treatment for this case may be found, for example, in Murray [2], while a general reference for enzyme kinetics is Laidler and Bunting [3].

Recently Segal and Slemrod [4] critically re-examined a model biochemical reaction with a single intermediate and arrived at a dimensionless formulation of the problem containing a new, more general, small parameter. Their parameter not only includes the traditional one as a special case, but also allows the possibility of a singular perturbation approach to certain problems in which the initial ratio of enzyme to substrate concentration is no longer small. We refer to [4] for a survey of earlier results on the QSSA in the context of their new nondimensionalization. 
The purpose of this paper is to specifically illustrate the application of these ideas to a biochemical reaction with two intermediates where the old formulation is not applicable, that is, where the ratio of initial enzyme to substrate concentration is not small. Such problems appear to be particularly important in vivo, where often the latter ratio is order unity (Sols and Marcos [5]). We show that the new nondimensionalization and QSSA lead, under certain conditions, to simple analytic solutions for a reaction where the ratio of initial enzyme to substrate concentration may be any fixed positive number. The reaction we have chosen has two intermediate steps but the methods presented in this paper may also be applied to other reactions.

In Sect. 2 the reaction mechanism is discussed, and in Sect. 3 we nondimensionalize the system of ordinary differential equations describing the reaction, obtaining appropriate fast and slow timescales and the new small parameter. In Sect. 4 singular perturbation methods are used to study several different parameter regimes where the reaction exhibits different types of behaviour. We also compare our analytical results with numerical simulations of the full system. Finally, in Sect. 5 we discuss possible applications of our work to actual biochemical reactions, and draw some conclusions.

\section{The reaction mechanism}

We consider a biochemical reaction in which an enzyme (concentration $E$ ) reacts reversibly with a substrate (concentration $S$ ) to form an intermediate enzymesubstrate complex (concentration $X$ ). The complex $X$ is then irreversibly transformed into a second enzyme substrate complex (concentration $Y$ ), which in turn irreversibly breaks down into the original enzyme and an altered substrate molecule called the product (concentration $P$ ). Perhaps the best known example of an enzyme-catalysed reaction that takes place in two steps is the hydrolysis of proteins by proteolytic enzymes, such as chymotrypsin. This process (digestion, to the physiologist) takes place via an acylenzyme. In molecular terms, there are two intermediates: the first is a non-covalent complex, in that no covalent bonds are broken nor new ones formed. The changes involving the second intermediatethe acylenzyme-do entail some chemistry; here covalent bonds in the substrate are broken and new covalent bonds with the enzyme are formed. A great deal of effort is devoted to the study of such reactions. The general reaction scheme is of the form

$$
S+E \underset{k_{-1}}{\stackrel{k_{1}}{\rightleftharpoons}} X \underset{k_{-2}}{\stackrel{k_{2}}{\rightleftharpoons}} Y \underset{k_{-3}}{\stackrel{k_{3}}{\rightleftharpoons}} E+P
$$

where the positive constants $k_{i}$ are reaction rates. To illustrate the method of mathematical analysis, we shall restrict our study to a special case of $(G)$, namely the reaction scheme

$$
E+S \stackrel{k_{1}}{\underset{k_{-1}}{\rightleftharpoons}} X \stackrel{k_{2}}{\longrightarrow} Y \stackrel{k_{3}}{\longrightarrow} E+P .
$$

Here we have made the assumption that $k_{-2} Y$ and $k_{-3} P$ are small in comparison to the other reaction rates, that is, these steps are effectively irreversible. In some biochemical cases, this is a good approximation to reaction $(G)$. The differential 
equations describing this reaction (using the law of mass action) are

$$
\begin{aligned}
& d S / d t=-k_{1} S E+k_{-1} X \\
& d E / d t=-k_{1} S E+k_{-1} X+k_{3} Y \\
& d X / d t=k_{1} S E-k_{-1} X-k_{2} X \\
& d Y / d t=k_{2} X-k_{3} Y \\
& d P / d t=k_{3} Y .
\end{aligned}
$$

For initial conditions we shall take

$$
\begin{aligned}
& E(0)=E_{0}, \quad S(0)=S_{0}, \quad X(0)=0, \\
& Y(0)=0, \quad P(0)=0 .
\end{aligned}
$$

The system (1a, b, c, d, e) with initial conditions (2) implies the conservation equations

$$
E+X+Y=E_{0}, \quad S+X+Y+P=S_{0} .
$$

The QSSA may be applied to biochemical reactions described by systems of ordinary differential equations in which, after an initial rapid transient, one (or more) of the dependent variables may be regarded as being in steady state with respect to the instantaneous values of the other dependent variables. This means that the former variables, instead of appearing differentiated on the left hand side of a system like ( $1 a, b, c, d, e)$, may be approximated as algebraic functions of the latter variables. The occurrence of this phenomenon is suggested in the reaction above by experimental measurements of the combination $P+Y$ after a relatively short pre-steady state period but before the substrate concentration appreciably decays. Often the "reaction rate" (that is, the rate of formation of $P+Y$ ) is approximately constant over this time interval, so that by adding (1d) and (1e) it follows that $X$ is also approximately constant over the same time interval. Consequently the approximation $d X / d t \approx 0$ may be valid for some time. With this and (3a), one can solve (1c) for $X$ in terms of $S$ and $Y$, obtaining

$$
X=\left(E_{0}-Y\right) S /(K+S)
$$

where

$$
K \equiv\left(k_{-1}+k_{2}\right) / k_{1} .
$$

Equation (4) indicates that $X$ is in steady state with respect to the instantaneous values of $S$ and $Y$. The evolution of $S$ and $Y$ is determined by (1a) and (1d), but the heuristic argument above becomes difficult to carry further because of a lack of knowledge of the relative sizes of various terms in the equations. In some circumstances, $Y$ will be much smaller than $E_{0}$ and so may be neglected in (4). Then using (4) in (1a) yields

$$
d S / d t=-k_{2} E_{0} S /(K+S) .
$$

If we assume that the substrate concentration does not change appreciably during the pre-steady state period, then we may use the initial condition $S(0)=S_{0}$ 
together with (6) to determine $S$. When $Y$ is comparable to $E_{0}$, it is not clear how to proceed. Note, however, that $X$ must increase from its initial value of zero relatively rapidly during the pre-steady state period, since by adding (1d) and (1e), the rate of change of $P+Y$ is proportional to $X$. At this point nondimensionalization of the system is necessary to proceed systematically.

\section{Nondimensionalization: choice of scales}

By using (3a) to eliminate $E$ and noting that (1e) can be used to determine $P$ if $Y$ is known, it suffices to consider the problem

$$
\begin{aligned}
& d S / d t=-k_{1} S\left(E_{0}-X-Y\right)+k_{-1} X \\
& d X / d t=k_{1} S\left(E_{0}-X-Y\right)-\left(k_{-1}+k_{2}\right) X \\
& d Y / d t=k_{2} X-k_{3} Y . \\
& S(0)=S_{0}, X(0)=0, \quad Y(0)=0 .
\end{aligned}
$$

The choice of time scales is central to further development. An excellent description of scaling for problems involving QSSA can be found in [4]. For our purposes we note the following points: (1) dimensionless variables are obtained by dividing dimensional variables by their scales, the latter estimating a variable's maximum order to magnitude; (2) dimensionless variables (and their dimensionless derivatives) are of unit order of magnitude, so that relative orders of magnitude of terms in dimensionless equations can be decided by dimensionless parameters appearing in the equations; (3) scaling requires some prior knowledge of the solution; (4) different scalings may be required in different domains for the independent variables, and in different domains of parameter space. Further discussions of nondimensionalization of systems of ordinary differential equations may be found in Kevorkian and Cole [6], and Lin and Segel [7].

The QSSA will often be valid when the reaction under consideration can be thought of as being composed of two distinct periods, the pre-steady state and the steady state. During the pre-steady state "rapid" transients occur while the substrate concentration changes little. After this initial period of activity, the system may be described as in a steady state, some of the dependent variables being in instantaneous "equilibrium" with respect to others. Let us call $t_{p}$ and $t_{s}$ respectively the characteristic time scales for the pre-steady state and steady state periods. It is important to note that it is not the case that there will always be a $t_{p}$ and a $t_{s}$ scale, since this will depend on whether the system is open or closed, the initial conditions and the magnitude of the reaction rates. However, we shall restrict ourselves to cases where such scales do exist. If we let lower case letters denote dimensionless variables and $\tau$ denote the dimensionless time obtained by scaling the dimensional time $t$ by the characteristic pre-steady state time $t_{p}(\tau=$ $\left.t / t_{p}\right)$, then during the pre-steady state the dimensionless variables are functions of $\tau$. We should choose appropriate scales for the pre-steady state so that $d x / d \tau$ is of unit order of magnitude and $d s / d \tau \approx 0$, that is, so that the dimensionless time rate of change of $x$ is order one while that of the substrate $s$ is approximately zero. This is what we shall mean by the pre-steady state. 
On the other hand, during the second period, the steady state, time is scaled by $t_{s}$, and if we denote by $T$ the dimensionless time obtained by scaling the dimensional time $t$ by $t_{s}\left(T=t / t_{s}\right)$ so that steady state dependent variables are functions of $T$, then we should choose appropriate scales for the steady state so that $d s / d T$ is of unit order of magnitude indicating that substrate is being used up. Additionally, the dimensionless derivative $d x / d T$ should appear multiplied by a small parameter, indicating that $x$ is in a steady state with respect to the instantaneous values of the other dependent variables. By seeking separate asymptotic expansions for the dependent variables in each of the pre-steady state and steady state periods and using the method of matching, we can combine them to obtain a composite expansion which is uniformly valid over the time interval of interest.

A further necessary consistency requirement is that $t_{p} \ll t_{s}$; the pre-steady state period consists of rapid transients during which little substrate is consumed, and to ensure this it is not merely sufficient to have $d s / d t$ small during the pre-steady state. We must also require the duration of the pre-steady state $\left(t_{p}\right)$ to be much smaller than that of the steady state $\left(t_{s}\right)$.

The following method often works well in isolating appropriate scales in physical problems. We nondimensionalize the dependent and independent variables by letting

$$
S=\alpha s, \quad X=\lambda x, \quad Y=\pi y, \quad t=\gamma t,
$$

where $s, x$ and $y$ are dimensionless variables, $t$ is a dimensionless time, $\gamma$ is a characteristic scale appropriate to the period under consideration, and $\alpha, \lambda$ and $\pi$ are appropriate scales for the dependent variables. We will endeavour to choose $\alpha, \lambda$ and $\pi$ so that $s, x$ and $y$ are of unit order of magnitude and a $\gamma$ for each of the pre-steady and steady state periods such that the previous consistency requirements necessary for the validity of the QSSA are met. This will determine the scales.

Condition (8a) immediately yields $\alpha=S_{0}$, so $s(0)=1$, but (8b, c) give no information about $\lambda$ and $\pi$. We will employ, for both the pre-steady and steady states, a scaling consistent with QSSA which simplifies the appropriate equations [(7b) in the pre-steady state, (7a) in the steady state] the least, that is, which makes as many terms as possible in the relevant differential equation have the same order of magnitude, thus in effect yielding the richest equation. (This principle is sometimes referred to as Kruskal's principle of minimum simplification; see [4].) The latter scaling arises from the belief that since we have no a priori reason for favouring one term over another in the equation, the most interesting scaled equation (and asymptotic limit) will often result by retaining the most terms.

Consider first the pre-steady state phase. We must choose scales so that $d x / d t$ is of unit order of magnitude and $d s / d t$ is small. Using (9) with $\alpha=S_{0}$ gives for (7b), after division by $\lambda$,

$$
\gamma^{-1} d x / d t=\left(\left\{k_{1} E_{0} S_{0}\right\} / \lambda\right) s-\left(k_{1} S_{0} s+k_{-1}+k_{2}\right) x-\left(\left\{k_{1} S_{0} \pi\right\} / \lambda\right) s y .
$$

We find that we can make all the terms on the right-hand side of (10) have the 
same order of magnitude if

$$
\left(k_{1} E_{0} S_{0}\right) / \lambda=\left(k_{1} S_{0}+k_{-1}+k_{2}\right)=\left(k_{1} S_{0} \pi\right) / \lambda
$$

(where, since $s=0(1)$ and $s$ changes little from its initial value $s(0)=1$ during the pre-steady state, we have set $s=1$ in the second term on the right-hand side of $(10))$.

Equation (11) then implies

$$
\pi=E_{0}, \quad \lambda=\left(E_{0} S_{0}\right) /\left(S_{0}+K\right),
$$

where $K$ is given in (5).

It now follows from (10) that to make $d x / d t$ of unit order of magnitude we should choose $\gamma$ so that

$$
\gamma \equiv\left[k_{1}\left(S_{0}+K\right)\right]^{-1} .
$$

This defines the characteristic time scale for the pre-steady state, and so defining $t_{p} \equiv \gamma$, we have

$$
t_{p} \equiv\left[k_{1}\left(S_{0}+K\right)\right]^{-1} \text {. }
$$

With $\tau=t / t_{p}$, the scaling in $(12 \mathrm{a}, \mathrm{b})$ and $\alpha=S_{0}$, we obtain the following dimensionless problem for the pre-steady state:

$$
\begin{aligned}
d s / d \tau & =\varepsilon[-s+\{\sigma /(1+\sigma)\} s x+s y+\kappa x /\{(1+\kappa)(1+\sigma)\}] \\
d x / d \tau & =s-[\sigma /(1+\sigma)] s x-s y-x /(1+\sigma) \\
d y / d \tau & =\sigma x /\left[(1+\sigma)^{2}(1+\kappa)\right]-[\theta /(1+\sigma)] y \\
s(0) & =1, \quad y(0)=0, \quad x(0)=0
\end{aligned}
$$

where

$$
\varepsilon=E_{0} /\left(S_{0}+K\right), \quad \sigma=S_{0} / K, \quad \kappa=k_{-1} / k_{2}, \quad \theta=k_{3} /\left(k_{-1}+k_{2}\right) .
$$

By construction, $d x / d \tau$ is of unit order of magnitude during the pre-steady state. We now see that if, as argued earlier, the dimensionless time rate of change of substrate $d s / d \tau$ is also to be small during the pre-steady state, then from (14a) we must have $\varepsilon \ll 1$. The alternative possibility, that the terms in parentheses on the right hand side of (14a) nearly cancel with each other is unlikely since, during the pre-steady state, $s$ changes little from its initial value $s(0)=1$ while $x$ is changing with time. This conclusion suggests that the appropriate small parameter for the problem is $\varepsilon$ as given in (16), and indeed this is the new small parameter obtained by Segel and Slemrod [4], but arrived at in a slightly different way. The fact that we can make $d s / d \tau$ small by the choice of a single parameter after completely determining the scaling from (14b) lends credence to the applicability of the QSSA to this problem.

If the conventional small parameter for biochemical problems, $E_{0} / S_{0}$, satisfies the condition $E_{0} / S_{0} \ll 1$, then certainly $\varepsilon \ll 1$ as well. In the laboratory, in order to carry out accurate experiments, it is usually, but not always, the case that $E_{0} \ll S_{0}$. However, as noted in [4], $\varepsilon$ may be small even in cases when $E_{0} / S_{0}$ is of order unity. In this paper we shall specifically consider the case when $\varepsilon \ll 1$ 
and $E_{0} / S_{0}$ is an arbitrary fixed positive number, that is, we shall consider the limit process in which $E_{0} / S_{0}$ is fixed and $\varepsilon \rightarrow 0$. Since, from (16)

$$
\varepsilon=\left(E_{0} / S_{0}\right) /\left[1+\left(K / S_{0}\right)\right]=[\sigma /(1+\sigma)]\left(E_{0} / S_{0}\right),
$$

this means that we are considering the case for which $E_{0} / S_{0}$ is fixed and $\sigma=$ $\left(S_{0} / K\right) \rightarrow 0$. From $(17)$, we note that

$$
\sigma=\left(S_{0} / E_{0}\right) \varepsilon+O\left(\varepsilon^{2}\right) .
$$

One way to think about what $\sigma \ll 1$ means is the following. The parameter $K$, defined in (5), has the dimension of a concentration. In fact, it defines a characteristic concentration for the reaction under consideration. If the ratio of the initial substrate concentration $S_{0}$ to the characteristic concentration $K$ is small, then by (16) $\sigma \ll 1$. Since we regard $E_{0} / S_{0}$ as a fixed positive number, (18) implies that $\sigma$ and $\varepsilon$ are nearly proportional. Requiring the ratio $E_{0} / S_{0}$ to remain fixed, independent of $\varepsilon$, is not necessary - it is possible to regard $E_{0} / S_{0}$ as a function of $\varepsilon$ but this adds unnecessary complications. When $E_{0} / S_{0} \ll 1$, the traditional approach may be employed. In cases of interest here $E_{0} / S_{0}$ is bounded away from zero as $\varepsilon \rightarrow 0$. By keeping $E_{0} / S_{0}$ fixed and reducing $S_{0}$ to make $\sigma=S_{0} / K \ll 1$, it may even be possible to experimentally realize conditions where the QSSA might be valid. Note also that, since we are holding $E_{0} / S_{0}$ fixed and $\alpha=S_{0}$, $\pi=\left(E_{0} / S_{0}\right) S_{0}$ and $\lambda=\varepsilon S_{0}$, all dependent variables scale like $S_{0}$.

To completely determine the solution to the above problem we must specify values for the parameters $E_{0} / S_{0}, \kappa$ and $\theta$, given in (16). It may happen that there are interesting parameter regions where $\kappa$ and $\theta$ are tending to zero, to infinity or staying fixed as $\varepsilon$ (and $\sigma$ ) are tending to zero. It is important to realize that in the present state of asymptotic analysis the notion of an asymptotic expansion makes sense only in one variable, that is, one small parameter, so that if $\theta$, for example, is tending to zero as $\varepsilon \rightarrow 0$, then $\theta$ should be related to some positive power of $\varepsilon$ by what is known as a similarity variable, a fixed, order one constant which is independent of $\varepsilon$. This means that $\theta=r \varepsilon$ where $r$ and $\delta$ are fixed positive numbers which do not depend on $\varepsilon$. In a problem where there are several dimensionless parameters the number of possible similarity variable relationships can become large, though often certain ones naturally suggest themselves. We consider some of these later. We refer to Kevorkian and Cole [6] for a discussion of similarity variables in several applied mathematical problems.

Proceeding now to the steady state, we assume, as is usually the case, that only the time scale need be changed to obtain the dimensionless formulation for this period. Returning to the scales in (9), we recall that since substrate is being used up during the steady state we wish to choose $\gamma$ so that $d s / d t$ has unit order of magnitude. Using the fact that $E_{0} k_{1}=\varepsilon\left[k_{1} S_{0}+k_{-1}+k_{2}\right]$ (see (17) and the definition of $K$ in (5)) (7a) becomes, in dimensionless form,

$$
\gamma^{-1} d s / d t=\varepsilon\left[k_{1} S_{0}(x s-s(1-y))+k_{-1}(x-s(1-y))+k_{2}(s(y-1))\right] .
$$

The dimensionless variables $s, x$ and $y$ have unit order of magnitude, $\varepsilon \ll 1$ and $\sigma=\left(k_{1} S_{0}\right) /\left(k_{-1}+k_{2}\right) \ll 1$, so in order to retain as many terms as possible on the right-hand side of equation (19) and to simultaneously make $d s / d t$ of unit order 
of magnitude we choose

$$
\gamma=\left[\varepsilon\left(k_{-1}+k_{2}\right)\right]^{-1} .
$$

As before, this defines the characteristic time scale $t_{s}$ for the steady-state

$$
t_{s} \equiv\left[\varepsilon\left(k_{-1}+k_{2}\right)\right]^{-1}
$$

It follows from (13) and (20) that

$$
t_{p} / t_{s}=\left[\varepsilon\left(k_{-1}+k_{2}\right)\right] /\left[k_{1}\left(S_{0}+K\right)\right]=\varepsilon /(1+\sigma)<\varepsilon,
$$

so that these time scales are consistent with the fact that the pre-steady state is of much shorter duration than the steady state. Defining $T=t / t_{s}$, and adapting the scaling in $(12 \mathrm{a}, \mathrm{b})$ (with $\left.\alpha=S_{0}\right)$ to nondimensionalize $(7 \mathrm{a}, \mathrm{b}, \mathrm{c}$ ), yields the following dimensionless system for the steady state,

$$
\begin{aligned}
d s / d T & =\sigma(x s-s(1-y))+[\kappa /(1+\kappa)] x-s(1-y) \\
\varepsilon d x / d T & =\sigma(s(1-y)-x s)+s(1-y)-x \\
\varepsilon d y / d T & =[\sigma /((1+\sigma)(1+\kappa))] x-\theta y,
\end{aligned}
$$

where $\varepsilon, \sigma, \kappa$ and $\theta$ are given in (16). Arbitrary constants appearing in the solution of $(22 a, b, c)$ are determined by matching to the solution for the pre-steady state, obtained from solving $(14 a, b, c),(15)$. We consider this in the next section.

\section{Approximate solutions}

We employ standard singular perturbation methods to obtain approximate solutions to the dimensionless systems obtained for the pre-steady and steady state periods. A solution of $(14 a, b, c)$ satisfying the initial conditions in (15) is sought in the form

$$
\begin{aligned}
& s(\tau)=s^{(0)}(\tau)+\varepsilon s^{(1)}(\tau)+\cdots \\
& x(\tau)=x^{(0)}(\tau)+\varepsilon x^{(1)}(\tau)+\cdots \\
& y(\tau)=y^{(0)}(\tau)+\varepsilon y^{(1)}(\tau)+\cdots
\end{aligned}
$$

From (18) and $\kappa /((1+\kappa)(1+\sigma))<1$, we find that regardless of the magnitude of the parameters $\theta$ and $\kappa$, the solution to the zeroth order problem for the pre-steady state is given by

$$
\begin{aligned}
& s^{(0)}(\tau)=1 \\
& x^{(0)}(\tau)=1-e^{-\tau} \\
& y^{(0)}(\tau)=0 .
\end{aligned}
$$

Recall that the dimensional dependent variables are given by

$$
S=S_{0} s, \quad X=\varepsilon S_{0} x, \quad Y=\left(E_{0} / S_{0}\right) S_{0} y,
$$


with $E_{0} / S_{0}$ fixed as $\varepsilon \rightarrow 0$, so that it is necessary to compute $y^{(1)}(\tau)$ to obtain the same order of magnitude information about $Y$ that (24b) yields about $X$. For $\kappa=0(1)$ and $\theta=0(1),(14 c),(15),(23 a, b, c)$ and $(24 a, b, c)$ give

$$
\begin{aligned}
d y^{(1)} / d \tau & =\left[S_{0} /\left(E_{0}(1+\kappa)\right)\right] x^{(0)}-\theta y^{(1)} \\
y^{(1)}(0) & =0 .
\end{aligned}
$$

When $\theta \neq 1$ and is bounded away from zero as $\varepsilon \rightarrow 0$ the solution to $(26 a, b)$ is

$$
y^{(1)}(\tau)=\left[S_{0} /\left(E_{0}(1+\kappa)\right)\right]\left[\left(1-e^{-\theta \tau}\right) / \theta-e^{-\tau}\left(\left(1-e^{-(\theta-1) \tau}\right) /(\theta-1)\right)\right],
$$

while if $\theta=1$ the solution is

$$
y^{(1)}(\tau)=\left[S_{0} /\left(E_{0}(1+\kappa)\right)\right]\left[1-e^{-\tau}-\tau e^{-\tau}\right] .
$$

Both (27) and (28) determine a final asymptotic value for $y^{(1)}(\tau)$ as $\tau \rightarrow \infty$ given by

$$
\lim _{\tau \rightarrow \infty} y_{1}(\tau)=S_{0} /\left(E_{0}(1+\kappa) \theta\right) .
$$

Note that increasing either $\kappa$ or $\theta$ reduces this value.

If, on the other hand, $\theta=0(\varepsilon)$ or $\theta=o(\varepsilon)$ as $\varepsilon \rightarrow 0$, then the solution is

$$
y^{(1)}(\tau)=\left[S_{0} /\left(E_{0}(1+\kappa)\right)\right]\left(\tau+e^{-\tau}-1\right) .
$$

We note that the dimensionless parameters $\varepsilon, \sigma, \kappa, \theta$ remain unchanged when $S_{0}$ and $E_{0}$ are multiplied by $B ; k_{-1}, k_{2}$ and $k_{3}$ are multiplied by $A$ and $k_{1}$ is multiplied by $A / B$, where $A$ and $B$ are arbitrary positive numbers. Consequently the choice of units used in the dimensional formulation may be changed by the scalings above without affecting the dimensionless formulation of the problem.

The linear growth in (30) indicates that the steady state value of $y$ in these cases is initially $0(1)$. We now verify this.

The solution of $(22 a, b, c)$, the steady state problem, is assumed to take the form

$$
\begin{aligned}
& s(T)=s_{0}(T)+\varepsilon s_{1}(T)+\cdots \\
& x(T)=x_{0}(T)+\varepsilon x_{1}(T)+\cdots \\
& y(T)=y_{0}(T)+\varepsilon y_{1}(T)+\cdots .
\end{aligned}
$$

At this point we must decide on the magnitudes of $\kappa$ and $\theta$ as $\varepsilon \rightarrow 0$. We shall consider four cases:

(i) $\theta=O(1), \kappa=O(1)$;

(ii) $\theta=o(\varepsilon), \kappa=O(1)$;

(iii) $\theta=O(\varepsilon), 1 /(\kappa+1)=O(\varepsilon)$;

(iv) $\theta=O(\varepsilon), \kappa=O(1)$. 
(i) $\theta=O(1), \kappa=O(1)$

The zeroth order problem, obtained by substituting $(31 \mathrm{a}, \mathrm{b}, \mathrm{c})$ into $(22 \mathrm{a}, \mathrm{b}, \mathrm{c})$ is

$$
\begin{aligned}
d s_{0} / d T & =[\kappa /(1+\kappa)] x_{0}-s_{0}\left(1-y_{0}\right) \\
0 & =s_{0}\left(1-y_{0}\right)-x_{0} \\
0 & =y_{0} .
\end{aligned}
$$

Equation (32b) represents the steady state relationship between $s_{0}, x_{0}$ and $y_{0}$ and is reminiscent of the dimensional relationship obtained heuristically in (4). In fact the two are the same for $\varepsilon \rightarrow 0$. Equations $(32 \mathrm{~b}, \mathrm{c})$ imply that $x_{0}=s_{0}$, and using this in $(32 a)$ gives

$$
\left.d s_{0} / d T=-[1+\kappa)\right] s_{0} .
$$

Solving (33) and using the matching condition between the pre-steady state and steady state solutions,

$$
\lim _{\tau \rightarrow \infty} s^{(0)}(\tau)=\lim _{T \rightarrow 0} s_{0}(T),
$$

to eliminate the arbitrary constant, we find

$$
\begin{aligned}
& s_{0}(T)=e^{-T /(1+\kappa)} \\
& x_{0}(T)=e^{-T /(1+\kappa)} \\
& y_{0}(T)=0 .
\end{aligned}
$$

The first-order term $y_{1}(T)$ is also easily obtained from $(22 \mathrm{c})$ when $\theta=O(1)$, and matching with the inner solution given by $(27),(28)$ yields

$$
y_{1}(T)=\left[S_{0} /\left(E_{0}(1+\kappa) \theta\right)\right] e^{-T /(1+\kappa)} .
$$

Note how the value of $\kappa$ determines the decay constant for $s_{0}$ and $x_{0}$. We may form a zeroth order composite solution, for example, $x_{\text {comp }}^{0}$, by adding the zeroth order pre-steady state and steady state solutions together and subtracting their common part - the limit obtained in the matching process above. The result is

$$
x_{\mathrm{comp}}^{0}(t)=e^{-t / t_{s}(1+\kappa)}-e^{-t / t_{p}} \text {. }
$$

For times $t$ such that $t \gg t_{p}$ and $t \ll t_{s}(1+\kappa), x_{\text {comp }}^{0}(t)$ is approximately constant, and hence so is the production of $P+Y$.

(ii) $\theta=o(\varepsilon), \kappa=O(1)$

For this case $\lim _{\varepsilon \rightarrow 0^{+}} \theta / \varepsilon=0$, so $\theta$ tends to zero faster than $\varepsilon$. The direction of the reaction from $Y$ to $E+P$ should be less favourable than in the previous case, and we will find $y_{0}(T) \neq 0$.

The pre-steady state solution is the same as before, $(24 a, b, c)$, while the zeroth order steady state problem is

$$
\begin{aligned}
d s_{0} / d T & =[\kappa /(1+\kappa)] x_{0}-s_{0}\left(1-y_{0}\right) \\
0 & =s_{0}\left(1-y_{0}\right)-x_{0} \\
d y_{0} / d T & =\left[S_{0} /\left(E_{0}(1+\kappa)\right)\right] x_{0} .
\end{aligned}
$$


Again (37a, b) imply

$$
d s_{0} / d T=[-1 /(1+\kappa)] x_{0}
$$

and combining this with $(37 \mathrm{c})$ gives

$$
\left(E_{0} / S_{0}\right) y_{0}+s_{0}=C,
$$

where $C$ is a constant. Matching as before to the pre-steady state solution implies $C=1$, so that

$$
y_{0}=\left[S_{0} / E_{0}\right]\left(1-s_{0}\right)
$$

Equations (37b), (38) and (40) then imply

$$
d s_{0} / d T=[-1 /(1+\kappa)]\left(\left(1-S_{0} / E_{0}\right) s_{0}+\left(S_{0} / E_{0}\right) s_{0}^{2}\right) .
$$

Solving (41) and matching with the pre-steady state solution then yields

$$
\begin{array}{ll}
s_{0}(T)=\left[1-S_{0} / E_{0}\right] /\left\{\exp \left(\left(1-S_{0} / E_{0}\right) T /(1+\kappa)\right)-S_{0} / E_{0}\right\}, & \left(S_{0} / E_{0} \neq 1\right) \\
s_{0}(T)=1 /\{[T /(1+\kappa)]+1\}, & \left(S_{0} / E_{0}=1\right)
\end{array}
$$

Note that $\lim _{T \rightarrow \infty} s_{0}(T)=0$ for $E_{0} \geqslant S_{0}$, while $\lim _{T \rightarrow \infty} s_{0}(T)=1-E_{0} / S_{0}>0$ for $E_{0}<S_{0}$. From (38) and (40) it also follows that

$$
\begin{aligned}
x_{0}(T)= & \left\{\left(1-S_{0} / E_{0}\right)^{2} \exp \left(\left(1-S_{0} / E_{0}\right) T /(1+\kappa)\right)\right\} /\left\{\left(\operatorname { e x p } \left(\left(1-S_{0} / E_{0}\right) T /(1+\kappa)\right.\right.\right. \\
& \left.\left.-S_{0} / E_{0}\right)^{2}\right\}, \\
y_{0}(T)= & \left(S_{0} / E_{0}\right)\left\{\exp \left(\left(1-S_{0} / E_{0}\right) T /(1+\kappa)\right)-1\right\} /\left\{\operatorname { e x p } \left(\left(1-S_{0} / E_{0}\right) T /(1+\kappa)\right.\right. \\
& \left.\left.-S_{0} / E_{0}\right)\right\},
\end{aligned}
$$

for $S_{0} / E_{0} \neq 1$, while

$$
\begin{aligned}
& x_{0}(T)=1 /\left\{(T /(1+\kappa)+1)^{2}\right\} \\
& y_{0}(T)=T /\{(1+\kappa)+T\}
\end{aligned}
$$

for $S_{0} / E_{0}=1$. As before, the zeroth order composite solutions for $s, x$, and $y$ may be constructed by adding the pre-steady state and steady state solutions and subtracting the common parts. Note, in particular, that by matching (43b) and (30) and subtracting the common part, it follows that the zeroth order composite solution for $y$ is given by $(43 b)$.

(iii) $\theta=O(\varepsilon), 1 /(1+\kappa)=O(\varepsilon)$

Introducing the $\varepsilon$-independent similarity variables $r_{1}$ and $r_{2}$, we have

$$
\theta=r_{1} \varepsilon, \quad 1 /(1+\kappa)=r_{2} \varepsilon .
$$

Since $\kappa /(1+\kappa)=1-1 /(1+\kappa)=1+O(\varepsilon)$, the zeroth order problem for the steady state given by $(22 a, b, c)$ and $(31 a, b, c)$ is

$$
\begin{aligned}
d s_{0} / d T & =x_{0}-s_{0}\left(1-y_{0}\right) \\
0 & =s_{0}\left(1-y_{0}\right)-x_{0} \\
d y_{0} / d T & =-r_{1} y_{0} .
\end{aligned}
$$


The arbitrary constants appearing in the solution of $(46 a, b, c)$ are determined by matching with the pre-steady state solution $(24 a, b, c)$, yielding

$$
\begin{aligned}
& s_{0}(T)=1 \\
& x_{0}(T)=1 \\
& y_{0}(T)=0 .
\end{aligned}
$$

Thus the large size of $\kappa$ prevents substrate from being consumed. Equations $(47 \mathrm{a}, \mathrm{b}, \mathrm{c})$ follow formally from $(35 \mathrm{a}, \mathrm{b}, \mathrm{c})$ with $1 /(1+\kappa)=O(\varepsilon)$, and suggest a longer time scale $t_{s}(1+\kappa)$ for the steady state problem when $\kappa$ is very large. On this timescale, the solution is

$$
\begin{aligned}
& s_{0}=e^{-T_{s}} \\
& x_{0}=e^{-T_{s}} \\
& y_{0}=0,
\end{aligned}
$$

where $T_{s}=t_{s}(1+\kappa)$. Note that this solution is the same as that for case (i).

(iv) $\theta=O(\varepsilon), \kappa=O(1)$

Again we introduce the similarity variable $r$ defined by $\theta=r \varepsilon, r$ being independent of $\varepsilon$. The zeroth order problem follows from $(22 \mathrm{a}, \mathrm{b}, \mathrm{c}),(31 \mathrm{a}, \mathrm{b}, \mathrm{c})$ :

$$
\begin{aligned}
d s_{0} / d T & =[\kappa /(1+\kappa)] x_{0}-s_{0}\left(1-y_{0}\right) \\
0 & =s_{0}\left(1-y_{0}\right)-x_{0} \\
d y_{0} / d T & =\left[S_{0} /\left(E_{0}(1+\kappa)\right)\right] x_{0}-r y_{0}
\end{aligned}
$$

A phase plane argument indicates $s_{0} \geqslant 0, x_{0} \geqslant 0$ and $y_{0} \geqslant 0$; from (48a) it follows that

$$
d / d T\left[\ln s_{0}\right]=-[1 /(1+\kappa)]\left(1-y_{0}\right)
$$

Differentiating (49) again gives

$$
\begin{aligned}
d^{2} / d T^{2}\left[\ln s_{0}\right] & =[1 /(1+\kappa)]\left(d y_{0} / d T\right) \\
& =[1 /(1+\kappa)]\left(S_{0} /\left(E_{0}(1+\kappa)\right) x_{0}-r y_{0}\right]
\end{aligned}
$$

Using $(48 \mathrm{a}, \mathrm{b})$ then implies

$$
d^{2} / d T^{2}\left[\ln s_{0}\right]=-\left(S_{0} / E_{0}(1+\kappa)\right) d s_{0} / d T-r\left\{\left(d / d T \ln s_{0}\right)+1 /(1+\kappa)\right\} .
$$

Integrating (51) once, matching with the pre-steady state solution and letting

$$
s_{0}=e^{F(T)}, \quad F(0)=0
$$

finally gives

$$
d F / d T+\left[S_{0} /\left(E_{0}(1+\kappa)\right)\right] e^{F}+r F+(1 /(1+\kappa))\left(r T+1-S_{0} / E_{0}\right)=0 .
$$



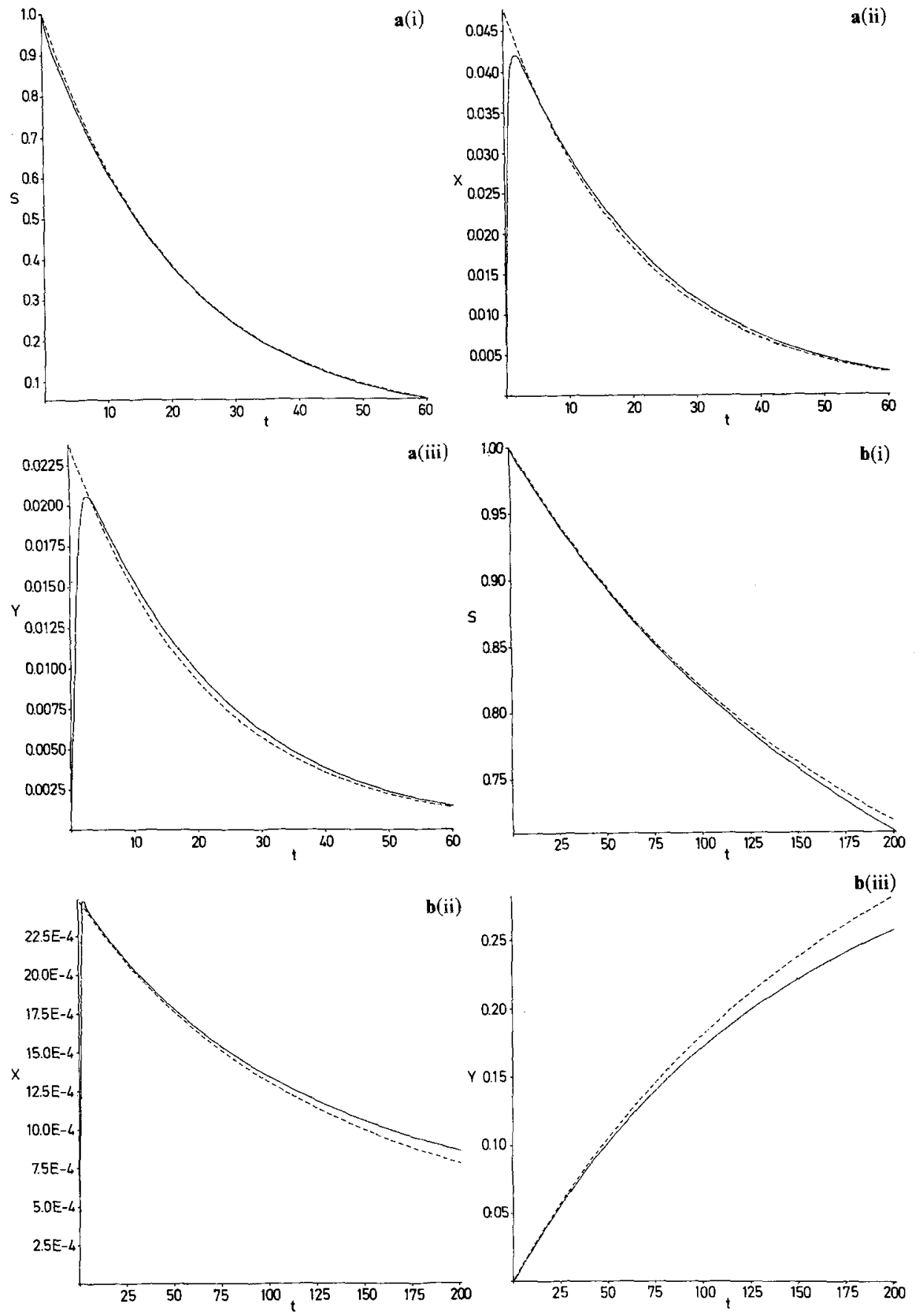

Fig. 1. a Comparison of numerical solution (solid line) of the full system (7), (8) with the analytical approximation (dashed line) for the outer timescale $t_{s}$ for parameters $k_{1}=0.1, k_{2}=1, k_{3}=2, k_{-1}=1$, $S_{0}=E_{0}=1$. Here, $\varepsilon=0.047619, \theta=1, \kappa=1, t_{s}=10.5$. This corresponds to case (i) in the text, and the analytical approximation is given by (35a), (35b) and (35d) (converted to dimensional form). b This corresponds to case (ii) with $E_{0} \neq S_{0}$. The analytical approximation is given by (42a), (43a) and (43b) (converted to dimensional form). Here, $k_{1}=0.01, k_{2}=1, k_{3}=0.001, k_{-1}=1, S_{0}=1, E_{0}=0.5$, $\varepsilon=0.0024876, \theta=0.0005, \kappa=1, t_{s}=201$. Parameter values have been chosen for purely illustrative purposes 
Equation (52) apparently cannot be solved in closed form. For given $S_{0} / E_{0}, \kappa$ and $r$ the solution of (52) with initial condition $F(0)=0$ can be determined numerically. Then

$$
\begin{aligned}
& s_{0}=e^{F} \\
& y_{0}=(1+\kappa) d F / d T+1 \\
& x_{0}=-(1+\kappa) e^{F} d F / d T .
\end{aligned}
$$

Numerical solutions of all the above cases compared very favourably with the analytical approximations. Figure 1 illustrates some of these. It must be noted that the parameter regimes wherein some of the above cases occur may be unrealistic from a biochemical viewpoint. However, the mathematical treatment is of intrinsic interest.

\section{Conclusion}

In this paper we have extended the parameter domain for which the quasi-steady state assumption may be applied to the reaction scheme

$$
E+S \underset{k_{-1}}{\stackrel{k_{1}}{\rightleftharpoons}} S \stackrel{k_{2}}{\longrightarrow} Y \stackrel{k_{3}}{\longrightarrow} E+P
$$

by using scaling arguments due to Segel and Slemrod [4]. Approximate solutions have been obtained for certain cases in which the ratio of initial enzyme to substrate concentration is a fixed positive number. Our analytical approximations compare favourably with numerical solutions of the full system.

Our results and methods might be useful for biochemical reactions in vivo, where often the ratio of initial enzyme to substrate concentration is order one; previous analytical work, valid only when the latter ratio is small, is not applicable to such problems. The analysis here, however, remains valid when the rate constants of the reaction and the initial substrate concentration satisfy a certain condition and predicts the time variation of chemical concentrations in terms of these rate constants. Open systems have added complications such as variable reaction rates, diffusion of chemicals, etc. The results and procedures discussed in this paper are a first step towards dealing with the more general case. We have shown that the behaviour of the system depends on the values of the parameters. In some parameter domains, $s$ decreases exponentially, in others, it decreases as a rational function (Fig. 1). Thus, by looking at the qualitative behaviour of the chemical concentrations in an experiment, one may be able to determine the parameter regime and thus fit the appropriate analytical approximation to the data. This would be a quicker and more reliable method for finding reaction rates than simply fitting the full ordinary differential equation system to the data. Consequently these results may also be used to evaluate reaction rates in some experiments.

We have shown that by decreasing the initial substrate concentration while keeping the ratio of initial enzyme to substrate concentration fixed, one can move into a parameter regime where the quasi-steady state assumption is valid. In this way perhaps one can modify experiments in order to apply the analytical results 
of Sect. 4. The ideas we have applied to reaction (R) can very likely be extended to other more complicated reaction schemes, such as $(\mathrm{G})$.

In a future publication we shall combine the analytical results obtained here together with experimental data to estimate the rates of reaction in a scheme like (R).

Acknowledgements. We would like to thank Professor J. D. Murray, Centre for Mathematical Biology, Oxford, and Dr. Stephen Waley, Dunn School of Pathology, Oxford, for several helpful discussions. C.L.F. would also like to thank Professor J. D. Murray for his hospitality during Michaelmas Term, 1987, while P.K.M. would like to acknowledge Science and Engineering Research Council of Great Britain Grant No. GR/C/63595.

\section{References}

1. Michaelis, L., Menton, M. L.: Die Kinetik der Invertinwirkung. Biochem. Z. 49, 333-69 (1913)

2. Murray, J. D.L Lectures on nonlinear-differential-equation models in biology. Oxford: 1977 Clarendon Press

3. Laidler, K. J., Bunting, P. S.; The chemical kinetics of enzyme action, Oxford: Clarendon Press 1973

4. Segel, L. A., Slemrod, M.: The quasi-steady state assumption: a case study in perturbation. SIAM Review (to appear)

5. Sols, A., Marco, R.: Concentrations of metabolites and binding sites. Implications in metabolic regulation. In: Horecker, B., Stadman E. (eds.) Current topics in cellular regulation, vol. 2, pp. 227-273 New York, Academic Press 1970

6. Kevorkian, J., Cole, J. D.: Perturbation methods in applied mathematics. Berlin Heidelberg New York: Springer 1981

7. Lin, C. C. Segel, L. A.: Mathematics applied to deterministic problems in the natural sciences. New York: Macmillan 1974

Received February 10/Revised June 7, 1988 